\title{
Spontaneous tumour lysis syndrome, as a presenting feature in a patient with Acute Lymphoblastic Leukemia
}

\author{
Mohit Mathur ${ }^{1}$,Sanjeev Sharma ${ }^{1}$ Rajesh Kumar ${ }^{1}$, Pankaj Beniwal ${ }^{1}$, \\ Dhananjai Agarwal ${ }^{1}$, Vinay Malhotra ${ }^{1}$ \\ ${ }^{I}$ Department of Nephrology, Sawai Man Singh Medical College \& Hospital, Jaipur. Rajasthan, India
}

\begin{abstract}
Tumour lysis syndrome (TLS) is a common problem in patients on treatment for malignancies; Spontaneous tumour lysis syndrome, however is a rare condition and should be suspected in patients with unexplained renal failure having typical biochemical abnormalities of hyperuricemia, hyperphosphatemia, hyperkalemia and hypocalcemia. It may sometimes be the first manifestation of an underling malignancy. Herein we present a case of spontaneous tumour lysis syndrome with Acute kidney injury, requiring hemodialysis, as the initial manifestation of Acute lymphoblastic leukemia (ALL). He responded well to hemodialysis and was able to tolerate the subsequent chemotherapy.
\end{abstract}

Key words: Spontaneous tumour lysis syndrome, hyperuricemia, hyperphosphatemia, hypocalcemia, Acute kidney injury

\section{Introduction:}

Tumour lysis syndrome is a life threatening condition and a true medical emergency ${ }^{[1][2][3]}{ }^{\text {It }}$ is one of the common causes of death in patients with hematological malignancies ${ }^{[1]}$ Prompt recognition and treatment is of paramount importance. However, few patients present with spontaneous tumour lysis syndrome ${ }^{[6]}$ and this may be the first manifestation of malignancy in these patients. These patients are deprived of the beneficial effects of proven preventive treatment strategies like aggressive hydration, allopurinol and rasburicase ${ }^{[1][2][3]}$

${ }^{\left[{ }^{4} \mathrm{We}\right.}$ are reporting a case of spontaneous tumour lysis syndrome with Acute kidney injury, requiring hemodialysis, as the initial manifestation of Acute lymphoblastic leukemia (ALL)

\section{Case Report:}

32 year old army recruit presented with the complaints of reduced urine output, nausea, anorexia and spontaneous subconjunctival hemorrhage since 5-7 days. He had been unwell for past 2-3 weeks, with complaints of low grade fever, petechial rashes over his chest, night sweats, anorexia and malaise. There was no history of seizures, syncope, palpitations, respiratory distess, cramps, tetany or altered sensorium prior to admission or during his stay in the hospital. Prior to admission he had taken paracetamol for fever and malaise. He had not sought any formal medical opinion prior to admission at our hospital.

On examination, he was pale and had mild edema. His vital signs were stable. He had few enlarged non-ender cervical lymphnodes. He had bilateral subconjunctival hemorrhage. He had petechial rashes over his chest. On abdominal examination, he had splenomegaly of about $4 \mathrm{cms}$ below the costal margin and mild hepatomegaly. His other systemic examination was unremarkable.

22,100/cumm.

His investigations (Table:1) revealed $\mathrm{Hb}$ of $5.6 \mathrm{gm} \%$, TLC of 67,000/cumm, Platelet count of

Peripheral smear showed absolute Lymphocytosis with few atypical cells.

LDH: $1450 \mathrm{IU} / \mathrm{L}$

Urine routine and microscopy: 4-6 RBC's/HPF, 8-10 WBC's/HPF; 2+ Proteinuria

Ultrasound of the kidneys revealed normal sized kidneys with poor cortico-medullary differentiation, clinical findings of hepatosplenomegaly were confirmed.

ECG \& Chest $\mathrm{X}$ ray was normal.

Bone marrow examination revealed a diagnosis of Acute lymphoblastic leukemia (ALL) L1 subtype. (Figure : 1) 


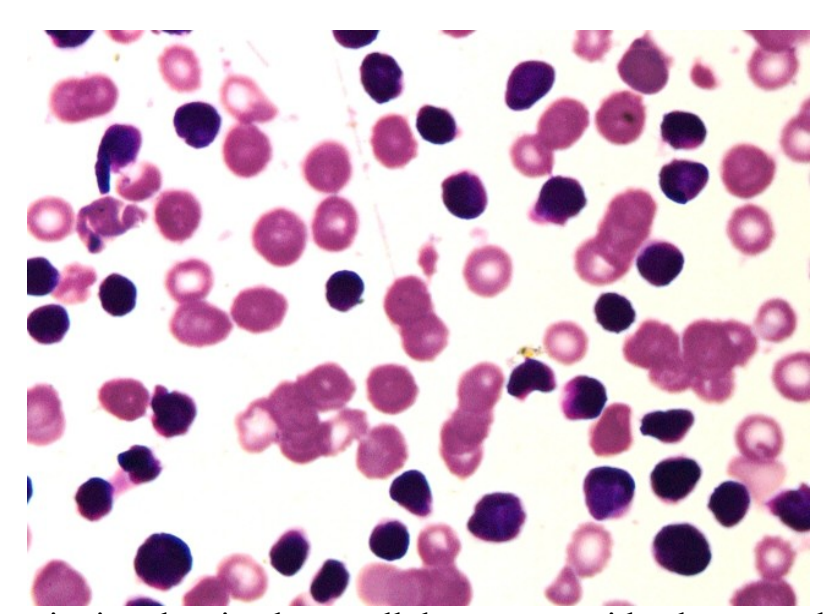

Figure:1 - Bonemarrow aspiration showing hypercellular marrow with a large number of lymphoid blast cells in a back ground of significantly depressed erythropoesis.

Immunohistochemistry was positive for CD-3, CD-5, CD-7, consistent with a diagnosis of $\mathrm{T}-$ cell Acute Lymphoblastic lymphoma.

In view of these findings, a diagnosis of Acute Kidney injury due to Spontaneous Tumour lysis syndrome was made in this patient with Acute lymphoblastic leukemia (ALL).

The patient was initiated on hemodialysis in view of the deranged renal functions, oliguria and uremic symptoms.

He received 4 sessions of hemodialysis over 7 days with improvement in biochemical parameters (Table: 1). Subsequently he was initiated on chemotherapy for ALL with vincristine, L-Asparginase and intra-thecal Methotrexate.

He received IV fluids, diuretics and low dose allopurinol along with other supportive treatment.

At the end of 2 weeks, he had good recovery of his renal functions with normalization of other biochemical parameters (Table: 1 )

\begin{tabular}{|l|l|l|l|l|l|}
\hline Parameter & At admission & Day 3 & Day 7 & Day 10 & Day 14 \\
\hline Sr. creatinine, $\mathrm{mg} / \mathrm{dl}$ & 8.3 & 6.4 & 3.0 & 2.2 & 1.24 \\
\hline Sr. Uric acid, mg/dl & 27 & 17 & 10.1 & 8.1 & 7.7 \\
\hline Sr. calcium, mg/dl & 7.0 & 7.2 & 7.8 & 7.88 & 8.1 \\
\hline Sr. phosphate, mg/dl & 8.3 & 7.9 & 6.8 & 6.20 & 5.12 \\
\hline Sr. potassium, mg/dl & 5.2 & 5.01 & 5.12 & 4.82 & 4.8 \\
\hline Urine output in 24 hours, $\mathrm{ml}$ & 500 & 600 & 1000 & 1500 & 2000 \\
\hline
\end{tabular}

(Table: 1)

\section{Discussion:}

In this patient, the presentation was predominantly with uremic symptoms of nausea, anorexia, malaise and oliguria., with complete recovery of renal functions at discharge.

The diagnosis of acute lymphoblastic leukemia (T cell ALL - L 1), associated with spontaneous tumor lysis syndrome was made upon investigation for the cause of acute kidney injury.

The triggering factor for Tumour lysis syndrome in this patient is unclear, as this patient had not received any form of chemotherapy and he did not fit in the category of high risk patients for TLS, as his initial WBC counts were $<2,00,000 /$ cumm. ${ }^{[2][3][4][5]}$

Tumour lysis syndrome (TLS) represents a set of metabolic complications that arise from treatment of a rapidly proliferating and drug-sensitive neoplasm, commonly occurring in hematological malignancies ${ }^{[1]}[2]$ ${ }^{[5}$ but also rarely associated with solid organ tumours also ${ }^{[7]}$. The incidence of TLS in all patients with malignancies ranges from $5 \%$ to $20 \%^{[1][2][3][4]}$. Spontaneous TLS is a rare condition, with only few case reports $^{[8][9][10]}$. There is no data on the incidence of spontaneous tumour lysis syndrome in patients with various malignancies. The mechanism of spontaneous tumour lysis syndrome remains unclear.

The Cairo-Bishop definition of TLS was proposed in $2004^{[1]}$ it provided specific laboratory criteria for the diagnosis of TLS. The same criteria is applicable to spontaneous TLS. 


\section{Cairo-Bishop diagnostic criteria for tumour lysis syndrome}

\section{Laboratory criteria (LTLS)}

- Uric acid $\geq 8 \mathrm{mg} / \mathrm{dl}$ or a $25 \%$ increase from baseline

- Phosphorus $\geq 4.5 \mathrm{mg} / \mathrm{dl}$ or a $25 \%$ increase from baseline

- Potassium $\geq 6.0 \mathrm{mmol} / \mathrm{L}$ or a $25 \%$ increase from baseline

- Corrected calcium $\leq 1.75 \mathrm{mmol} / \mathrm{L}$ or a $25 \%$ decrease from baseline

\section{Clinical criteria*(CLTLS)}

- Creatinine $\geq 1.5$ times the upper limit of normal

- Cardiac arrhythmia or sudden death

- Seizure

* May not be directly related to a therapeutic agent

\section{(Table: 2$)$}

Acute spontaneous TLS ${ }^{[1][2][6]}$ occurs prior to the initiation of cytotoxic therapies (including chemotherapy, radiotherapy and immunotherapy)

Spontaneous tumor lysis syndrome may be the first symptom of underlying malignancy, as in our patient. Spontaneous TLS also deprives the patient, the benefit of proven pre-treatment measures, such as hydration, allopurinol etc ${ }^{[1][2][3][4][5]}$ which has become the norm nowadays.

The metabolic complications of TLS predispose the cancer patient to clinical toxicities including renal insufficiency, cardiac arrhythmias, seizures, neurological complications and potentially sudden death ${ }^{[1][3]^{[4]}}$

Prior to chemotherapy or any other cytoreductive measures, all patients with malignancies should always be risk stratified ${ }^{[1][2][3][4]}$ as high risk, intermediate risk or low risk for TLS according to the type of underlying malignancy, cell burden (in leukemia and lymphoma), sensitivity to therapy, type of therapy planned (eg: chemotherapy, steroids, radiotheraoy etc), level of LDH above normal, presence of pre-existing: renal dysfunction, hyperuricemia, hyperphosphatemia and hypocalcemia ${ }^{[1][2][3][4][5]}$

TLS is a medical emergency and can be life threatening ${ }^{[1][2] . ~ T h e ~ g e n e r a l ~ p r i n c i p l e s ~ o f ~ t r e a t m e n t ~}$ remain same in both post chemotherapy TLS and spontaneous TLS. Low risk patients are treated with IV fluids alone, intermediate risk patients need IV hydration and allopurinol ${ }^{[1][2]}[3][4][5]$ High risk patients need aggressive hydration and rasburicase, ${ }^{[1]}{ }^{[4][5]}$ however rasburicase is not readily available at present.

Patients should be hydrated with approximately $3-4$ L/day, depending on the underlying renal and cardiac status. ${ }^{[1][2][3][5]}$ Diuretics may be added if necessary. Treatment of asymptomatic hypocalcaemia is generally not recommended, as the risk of precipitating metastatic calcification is high, especially in the setting of hyperphosphataemia ${ }^{[1]\left[2^{[2]}[3][4]\right.}$ Currently there is no role for alkalinisation of urine, oral phosphate binders and febuxostat ${ }^{[1][2][3][4]}$

Biochemical parameters like uric acid, electrolytes, calcium, phosphate, serum creatinine, LDH etc should be determined before therapy and every 4-6 hours for the first 48-72 hours after the initiation of antitumor therapy and regularly monitored thereafter ${ }^{[1][2][3][4] \text {. }}$

The old adage "Prevention is better than cure" is perhaps best exemplified in the management of TLS.

\section{References:}

[1]. Cairo MS, Bishop M. Tumor lysis syndrome: New therapeutic strategies and classification. Br J Haematology 2004;127:3-11

[2]. Coiffier, B., Altman, A., Pui, C.H., Younes, A. \& Cairo, M.S; Guidelines for the management of pediatric and adult tumor lysis syndrome: An evidence-based review. Journal of Clinical Oncology, 2008 ,26, 2767-2778

[3]. Scott C. Howard, M.D., Deborah P. Jones, M.D., and Ching-Hon Pui, M.D; The Tumor Lysis Syndrome:N Engl J Med 2011; 364:1844-1854

[4]. Cairo MS, Coiffier B, Reiter A, Younes A; TLS Expert Panel; Br J Haematology. 2010 May;149(4):578-86

[5]. Patrizia Tosi et al, Recommendations for the evaluation of risk and prophylaxis of tumour lysis syndrome (TLS) in adults and children with malignant diseases: an expert TLS panel consensus. Haematology December 1, 2008 vol. 93 no. 12 1877-1885

[6]. Jasek, A. M. and Day, H. J. Acute spontaneous tumor lysis syndrome. Am. J. Hematol., 1994, 47: 129-131

[7]. Gemici C, Tumor lysis syndrome in solid tumors. Clin Oncol 2006;18: 773-80.

[8]. Mateusz Opyrchal, Travis Figanbaum, Amit Ghosh, Vincent Rajkumar, and Sean Caples, Spontaneous Tumor Lysis Syndrome in the Setting of B-Cell Lymphoma, Case Reports in Medicine, vol. 2010, Article ID 610969, 3 pages, 2010

[9]. Samer alkhuja,Harry ulrich; Acute renal failure from spontaneous acute tumor lysis syndrome: A case report and review; Renal failure: 2002 24:2, 227-232

[10]. Sharma SK, Malhotra P, Kumar M, Sharma A, Varma N, Singh S; Spontaneous tumor lysis syndrome in acute lymphoblastic leukemia.: J Assoc Physicians India, 2005; 53:828 -30. 